\title{
CHARA and e-VLBI observations of Algol
}

\section{Zsolt Paragi}

JIVE, Dwingeloo, Netherlands

MTA Research Group for Physical Geodesy and Geodynamics, Penc, Hungary

E-mail: zparagi@jive.nl

\section{Szilárd Csizmadia}

Konkoly Observatory, Budapest, Hungary

E-mail: csizmadia@konkoly.hu

\section{Tamás Borkovits}

Baja Astronomical Observatory, Baja, Hungary

E-mail: borko@alcyone.bajaobs.hu

\section{László Mosoni}

Max Planck-Institut für Astronomie, Heidelberg, Germany

E-mail: mosoni@mpia.de

\section{László Sturmann}

CHARA, Georgia State Univ., USA

E-mail: sturmannechara-array.org

\section{Péter Ábrahám}

Konkoly Observatory, Budapest, Hungary

E-mail: abrahamekonkoly.hu

\section{Michael A. Garrett}

Astron, Dwingeloo, Netherlands

E-mail: garretteastron.nl

We present first results from long baseline optical and radio interferometry observations of the Algol system. We probe the close binary in these different regimes with comparable resolution (milliarcsecond scales) with the CHARA and the e-EVN arrays.

From planets to dark energy: the modern radio universe

October 1-5 2007

University of Manchester, Manchester, UK

*The CHARA Array is funded by the National Science Foundation through NSF grants AST-0307562 and AST06006958 and by Georgia State University through the College of Arts and Sciences and the Office of the Vice President for Research. e-VLBI developments in Europe are supported by the EC DG-INFSO funded Communication Network Developments project 'EXPReS', Contract No. 02662 (http://www.expres-eu.org/). The European VLBI Network (http://www.evlbi.org/) is a joint facility of European, Chinese, South African and other radio astronomy institutes funded by their national research councils. 

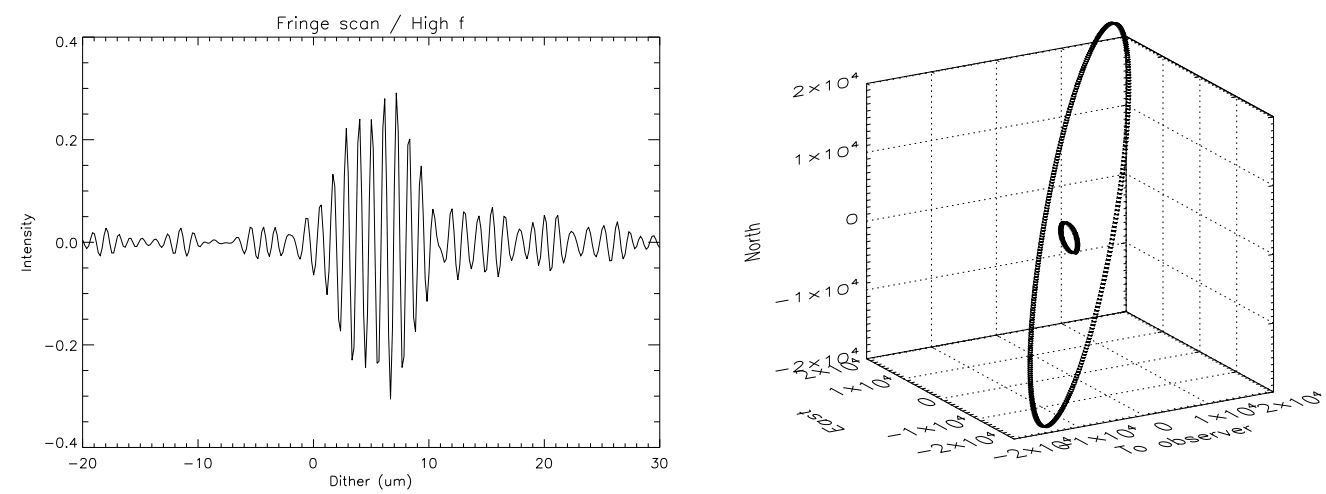

Figure 1: Left: an example CHARA fringe, filtered for noise. Right: geometry of the Algol system, obtained by model-fitting of the CHARA data. Note that the orbits are not to scale: the close binary orbit is 10.000 times smaller than shown.

\section{The Algol system}

Algol is an eclipsing binary stellar system in which the cool K2 IV sub-giant looses matter through Roche-lobe overflow to the more massive B8 V star. A third star is orbiting around the close binary in a long period orbit. The system is only 29 parsec away from us, being the nearest and one of the most active of its kind. Its proximity makes Algol an ideal target for optical and radio interferometric observations. The angular separation of the close binary is about 2 milliarcseconds, and the apparent size of the stars themselves is in the order of a milliarcsecond. With high resolution optical interferometry observation one may address the well-known three-body dynamical problem [1], while radio interferometry observations give information about the chromospheric activity of the cool companion.

\section{Observations and data reduction}

The CHARA Interferometer [2] observations took place on three consecutive nights on 1-3 December 2006. The array consists of six 1-m optical telescopes, any two of these can be used for interferometry. In total, 24 individual data points were collected from Algol at different phases, and 6-6 calibration measurements from HD 16895 and HD 19373. Raw data have been cleaned for atmospheric effects by applying low-frequency and high-frequency filters; as a result, good quality fringes were obtained. The fringe amplitudes (visibilities) have been calibrated using HD 16895 and HD 19373, that have accurate distance and radius measurements from HIPPARCOS.

Radio observations took place at $5 \mathrm{GHz}$ on 14 December 2006 for about 10 hours with six telescopes of the European VLBI Network (EVN): Cambridge, Jodrell Bank, Medicina, Torun, Onsala and Westerbork. Data were streamed at $256 \mathrm{Mbps}$ rate from each telescopes and correlated real-time at JIVE, a technique called e-VLBI. Algol was phase-referenced to 0309+411, a nearby compact calibrator. Synthesis array data from Westerbork have been analysed as well. We used 3C84 for D-term, and 3C138 for amplitude calibration. 


\section{CHARA results}

Because of the short orbital period of Algol (2.9 days), interpretation of the visibility curve has not been straightforward. One needs to sample various spatial frequencies as the projected baseline changes during the observations. In our case however the binary separation was changing as well, making robust model-fitting difficult.

The model was developed in the following way. First we assumed that the orbit is circular and it is characterized by the semi-major axis, the inclination and the argument of the ascending node. The shape of the components is determined by the Roche-model which requires three free parameters: the mass ratio (q) and the surface potentials of the two stars $\left(\Omega_{1}, \Omega_{2}\right.$ [3]). The intensity ratio of the two stars is a free parameter and the surface brightness distribution is modified by the gravity darkening and the limb-darkening. The gravity darkening exponents were chosen as usual and the limb darkening coefficients were taken from [4]. To determine the orbital phase, the times of minima were collected and were fitted by a polynomial to have a precise prediction of the conjuction. Finally, the two-dimensional projected picture of Algol was determined for each orbital phase where we had measurements. For this purpose the position angle of projected vector of the normal vector of the orbit was also changed (as one can see this position angle is equal to the node angle of the orbit). Then a grid search within reasonable limits of the parameters yielded the results.

This way we determined the orbit of the close pair. The projected maximum angular distance of Algol is $2.50 \pm 0.04$ mas which — from the spectroscopically known size of the semi-major axis - yields a distance of $26.1 \pm 0.4$ parsec for Algol. HIPPARCOS measured it to be $28.5 \pm 0.7$ parsec. These values are in agreement with each other within $3 \sigma$ limit. The argument of the node was found to be $49 \pm 3$ degrees in agreement with the earlier radio measurement of $52 \pm 5$ degrees [5]. In this case the orbital plane of the close pair and the third body has a mutual inclination of 84 degrees. This mutual inclination would produce significantly slower orbital precession rate than what is implied by [5] (mutual inclination of 100 degrees), however, the resulting period is still too short. Consequently, the variation of the inclination and eclipse depth would be fast, which is clearly in contradiction with the observations.

\section{4. e-EVN results}

The main goal of the e-EVN observations were to monitor the cromospheric activity of the system during secondary minima, when the B star eclipses the K subgiant. Although the resolution ( $\sim 6$ mas) was not enough to resolve the gyro-synchrotron lobes, a shift in the centroid of the radio image (after correcting for orbital motion) and a change in the circular polarisation properties would have hinted on the geometry of the radio-emitting lobes with respect to the subgiant.

The orbital motion of the K-star during the 10 hours observations was clearly detected. We attempted to use these data to further constrain the binary orbit, but for various reasons we could not obtain an reliable fit to the data. Algol went through a major flare during the observations, its flux density changed from about 50 to $10 \mathrm{mJy}$ in a couple of hours time. While this flare ruined our original goals, there was the opportunity to study the evolution of this event. Both the Westerbork synthesis and the e-VLBI data confirmed that the circular polarisation varied during 

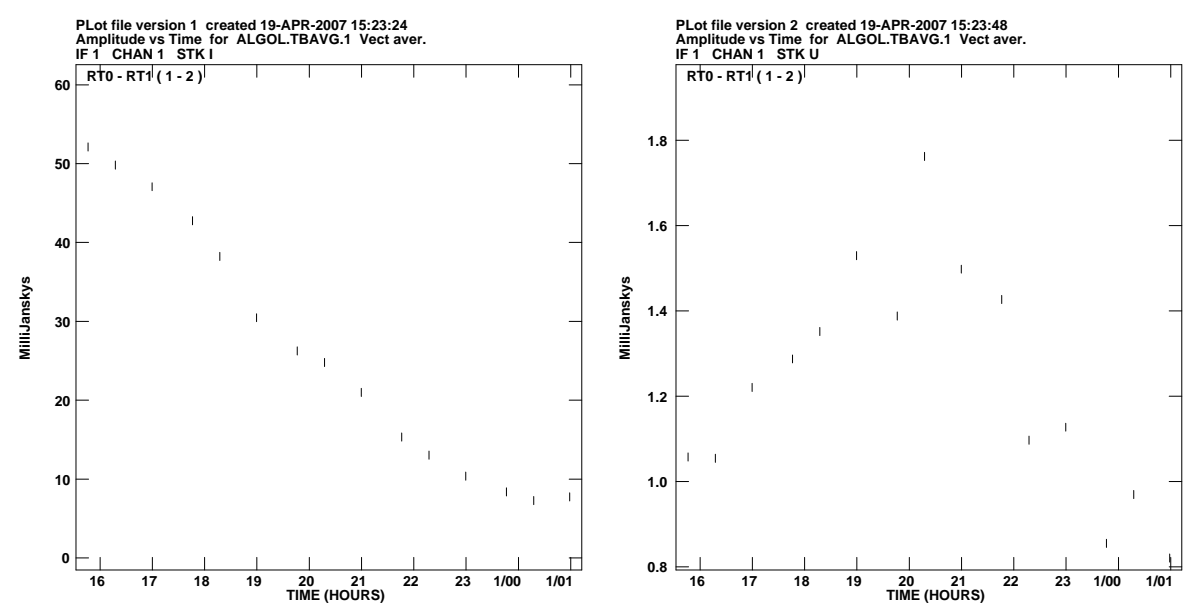

Figure 2: Left: radio lightcurve of Algol during the 14 December 2006 e-EVN observations, obtained by the Westerbork synthesis radio telescope. Right: circular polarisation variation in the same experiment. The e-EVN data were in agreement with the WSRT observations of the CP emission.

the outburst, and the fractional $\mathrm{CP}$ decreased significantly - a fact that has been known from previous studies (see [6] and references therein). Snapshot e-VLBI imaging of Stokes I and Stokes $\mathrm{V}$ data has shown that the circular polarisation and the total intensity flux centroids were off by a very significant amount of 2-3 milliarcseconds. This indicated that not only the flare had different characteristics than the well-known radio lobes, but it originated from a different location as well. An alternative explanation is that the flare occured only in one of the lobes.

\section{Conclusions}

Joint optical and radio interferometry observations are useful to study different aspects of bright, active stellar systems. With the CHARA data we were able to improve on the distance and the orbital parameters of Algol, but the dynamical problem remains open. Future higher resolution astrometric VLBI observation would be helpful to resolve these, as well as to locate the origin of the radio activity.

\section{References}

[1] T. Borkovits, E. Forgács-Dajka, Zs. Regály, Astron. Astrophys. 426, 951 (2004).

[2] T.A. ten Brummelaar, H.A. McAlister, S.T. Ridgway, W.G. Bagnuolo, Jr., N.H. Turner et al., Astrophys. J. 628, 453 (2005).

[3] Z. Kopal, Dynamics of Close Binaries, University of Drodrecht (1978)

[4] W. van Hamme Astron. J. 106, 2096 (1993).

[5] J.-F. Lestrade, R.B. Phillips, M.H. Hodges, R.A. Preston, Astrophys. J. 410, 808 (1993)

[6] R.L. Mutel, L.A. Molnar, E.B. Waltman, F.D. Ghigo, Astrophys. J. 507, 371 (1998) 